\title{
A New Approach to Percutaneously Treat Chronic Coronary Total Occlusions: The 'Minimalistic Hybrid Approach’ Algorithm
}

Percutaneous recanalisation of coronary chronic total occlusions has traditionally relied upon large-bore introducer sheaths and dual arterial access, with interventions frequently performed via the transfemoral approach. In what seems to be a paradigm shift to a new approach, the Editor's Pick article for this year's issue of EMJ Interventional Cardiology by Vescovo et al., describes the 'minimalistic hybrid algorithm' as an alternative procedure to the traditional methods. The authors discuss the choice of starting strategy, which takes into account the type of collaterals (ipsilateral or contralateral) and angiographical features of the lesion. Additionally, the review considers the efficacy and safety of this novel approach, recently assessed in one retrospective and one prospective single-centre study.

Authors: Giovanni Maria Vescovo, Carlo Zivelonghi, Benjamin Scott, Adriaan Wilgenhof, Yannick Willemen, Carl Convens, Stefan Verheye, Paul Vermeersch, *Pierfrancesco Agostoni

HartCentrum, Ziekenhuis Netwerk Antwerpen Middelheim, Antwerp, Belgium *Correspondence to agostonipf@gmail.com

Disclosure: The authors have declared no conflicts of interest.

Received: 02.03.21

Accepted: 14.05.21

Keywords: Angina, chronic coronary total occlusion (СTO), hybrid algorithm, minimalistic hybrid approach algorithm (MHA).

Citation: EMJ Int Cardiol. 2021;9[1]:51-57.

\section{Abstract}

Vascular access-related complications are one of the most frequent issues following percutaneous interventions of chronic total occlusions (CTO) because of the traditional use of large introducer sheaths for more back-up catheter support, and the need for multiple access sites, often including femoral access. In current practice, many operators still approach the revascularisation of CTO through femoral access despite the demonstrated advantage of radial procedures, mostly in terms of incidence of bleeding complications. Recently, an alternative strategy to deal with CTO, with the aim to minimise the number of access-related complications without affecting the successful revascularisation rate, has been proposed: the 'minimalistic hybrid approach' algorithm. This approach consists of the use of classic 'hybrid algorithm' techniques, but also aims to minimise the number of access sites, the size of the catheters used, and the adoption of the femoral access. 


\section{INTRODUCTION}

Percutaneous treatment of chronic total occlusion (CTO) is considered one of the most challenging procedures due to the high incidence of complications and the relatively low success rate compared with non-CTO percutaneous coronary interventions $(\mathrm{PCl}))^{1-3}$ However, the technical advances of the last two decades, the greater knowledge and experience shared by expert CTO operators, and the introduction of specific algorithms for treatment standardisation have led to higher procedural success, especially in high-volume CTO-PCl centres. ${ }^{4-7}$ Special attention was also given to the unsolved problem of vascular access-related complications because of the routine use of large-bore catheters and the insertion of multiple sheaths, often at the level of the femoral artery. Recently, a modified version of the classic 'hybrid algorithm', called the 'minimalistic hybrid approach (MHA) algorithm', was proposed with the aim to limit vascular complications without affecting the rate of successful revascularisation. ${ }^{8}$ This strategy consists of the use of classic hybrid algorithm techniques, but aims to minimise the number of access sites, the size of the catheters used, and the adoption of the femoral approach.

\section{CHRONIC TOTAL OCCLUSION REVASCULARISATION TECHNIQUES}

Four main techniques are commonly used during CTO revascularisation. These are defined as 'antegrade' or 'retrograde' strategies, according to the direction the lesion is crossed (from the proximal to the distal part and from the distal to the proximal part, respectively), and as 'wire escalation' or 'dissection and re-entry' techniques, according to guidewire location during СTO crossing (intraluminal or subintimal, respectively). ${ }^{9}$ Thus, it is possible to distinguish the strategies as follows: antegrade wire escalation (AWE); retrograde wire escalation (RWE); antegrade dissection and re-entry (ADR); and retrograde dissection and re-entry (RDR).

During wire escalation, the operator attempts to cross the CTO while remaining in the true lumen of the vessel. During the attempt, guidewires with higher tip stiffness and more penetration force are used progressively. In the dissection and reentry techniques, the guidewire is advanced through the subintimal space and redirected into the true lumen of the vessel once beyond the CTO lesion.

Finally, retrograde techniques are adopted in case of failure of antegrade options or when complex anatomy and a well-developed collateral circulation is present. ${ }^{10}$ Collateral circulation consists of a communication between two vessels (contralateral collaterals) or between two segments of the same vessel (ipsilateral collaterals) and represents an alternative source of blood flow for the myocardial area perfused by the CTO target vessel." These collaterals are traditionally used by interventional cardiologists during the procedure for obtaining an appropriate view of the coronary anatomy and to reach the distal cap of the occlusion. Guidewires and microcatheters are advanced through collaterals to gain access to the distal lumen of the occluded vessel..$^{2}$

\section{THE 'HYBRID ALGORITHM'}

The percutaneous treatment of CTO is associated with a substantial rate of complications. A recent prospective study in CTO-PCI suggested that the percentage of periprocedural complications is considerably greater compared to non-CTO-PCI, with an overall rate of in-hospital major adverse cardiac and cerebrovascular events of $7 \% .^{13}$ In particular, clinically relevant coronary perforation was found to be the most common event (4.8\%), followed by periprocedural myocardial infarction (2.6\%), death (0.9\%), and urgent surgery $(0.7 \%)$. Interestingly, no strokes were recorded among the 1,000 patients included in the registry. However, access site haematoma was seen in $4.3 \%$ of the patients, confirming a high rate of vascular-related complications in today's practice. Despite the fact that these rates are still high in the current era, significant improvements have been made over the past years. In particular, the development of $\mathrm{PCl}-\mathrm{CTO}$ algorithms has contributed to a decrease in the number of complications as well as an increase in the rate of successful revascularisation. ${ }^{6}$

One of the most commonly adopted algorithms, mainly across North America and Europe, is the so-called 'hybrid algorithm', which was proposed by a group of expert CTO operators in $2011 .{ }^{5}$ One of the key elements of this algorithm is the use of the dual injection technique that consists of 
the simultaneous engagement and injection of the coronary arteries to improve visualisation of the entire coronary anatomy. ${ }^{14}$ More specifically, a first injection is performed at the level of the vessel that provides collaterals (donor vessel), followed 2-3 seconds later by a contralateral injection in the target CTO vessel. Doing so, the contrast reaches the proximal and distal segment of the CTO at the same time and allows visualisation of the entire course of the occluded coronary artery.

One of the main advantages of this technique is that it allows understanding of the exact location of the guidewire, particularly once the body of the CTO is passed. In fact, without the injection from the donor vessel, in many cases it would not be possible to identify the segment beyond the СTO and therefore to discern if the guidewire is inside or outside the lumen of the vessel, with subsequent potential increased risk of complications such as perforation. The dual injection represents the first step of the procedure, allowing the evaluation of the following four anatomical characteristics: the ambiguity of the proximal cap; disease on the distal target (diffuse or not diffuse); the presence of favourable collaterals; and lesion length $(<20$ $\mathrm{mm}$ ). Subsequently, the operator can choose the starting strategy among the four aforementioned techniques, but with the possibility during the procedure to quickly switch from one strategy to another because of the presence of a guiding catheter in each coronary artery.

However, despite several advantages provided by the hybrid algorithm, a potential drawback may be represented by the systematic use of the simultaneous double injection. In fact, this requires two sites of access, with an increased chance of vascular-related complications. For this reason, a modified version of the classic hybrid algorithm, the MHA algorithm, has been proposed.8,15

\section{THE 'MINIMALISTIC HYBRID APPROACH' ALGORITHM}

The MHA algorithm has been developed with the aim to minimise the number of access sites as well as the size of the introducer sheath. Moreover, the use of forearm (radial/ulnar/ distal radial) access is strongly recommended over the femoral. In fact, despite the lower risk of vascular access-related complications with trans-radial approach, femoral access remains common in CTO-PCl practice; ${ }^{16}$ the reason being the complexity of CTO-PCl, which often requires large catheters (7-8 French $[\mathrm{Fr}]$ ). The main difference between the two algorithms is the use of simultaneous double injection. In the hybrid algorithm, dual injection is mandatory and considered a cornerstone because allowing an optimal visualisation of the entire target vessel (even the distal segment beyond the CTO) seems to make the procedure safer and increase the chance of successful revascularisation. The simultaneous visualisation of the entire vessel acknowledges the relationship between the proximal and the distal cap of the lesion, allowing the operator to point the guidewire towards the proper direction. This is particularly useful when wire escalation techniques with high-penetrationforce guidewires are used.

As mentioned, the aim of the MHA is to reduce the number of access sites and to minimise the size of the sheaths, in order to decrease the rate of vascular complications. To do so, the authors suggest choosing the access according to five different scenarios the operator may deal with. The initial set-up in all cases is represented by a single 6 or $7 \mathrm{Fr}$ sheath (trans-wrist). Thus, simultaneous double injection is not considered unavoidable in this algorithm.

The MHA recommends a careful evaluation of the anatomy in order to plan the procedure and to choose the best technique for each specific scenario. Performing a good coronary angiography in the procedural planning using the MHA is even more important than in the classic hybrid algorithm due to the lack of simultaneous dual injection during the procedure. Very long films performed by a single catheter allow analysis of the collateral circulation and the characteristics of the CTO. When in doubt, a 'stepwise double injection' is strongly recommended before starting the $\mathrm{PCl}$, with sequential assessment of the donor artery first and the CTO artery afterwards using one single arterial access site.

Using the angiographical features, the type of collaterals, and the technical preference of the operator, it is possible to choose between five different approaches (Figure 1). 


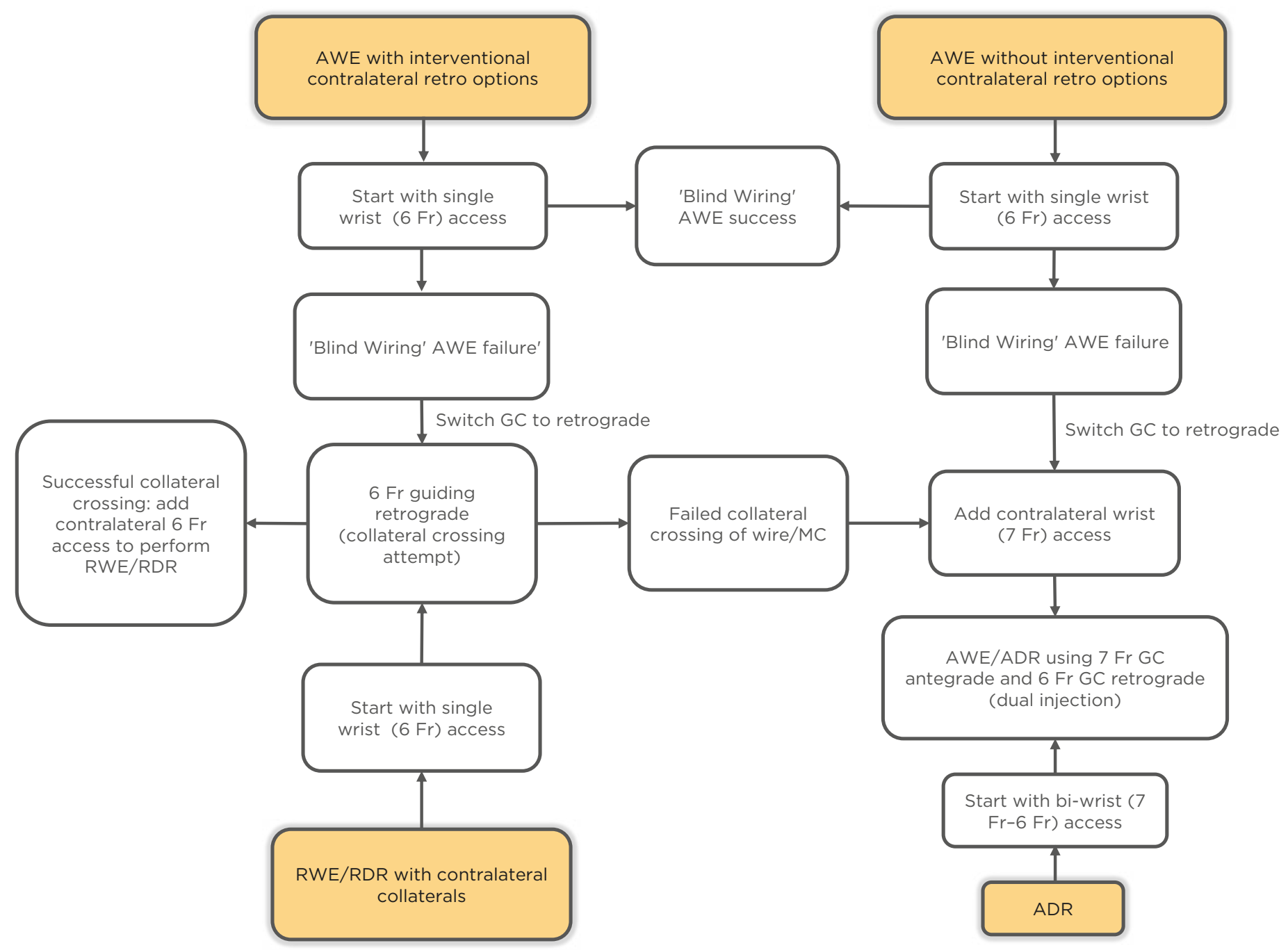

Figure 1: ‘Minimalistic hybrid approach’ algorithm flowchart.

Choice of set-up according to the starting strategy.

ADR: antegrade dissection and re-entry; AWE: antegrade wire escalation; Fr: French; GC: guiding catheter; MC: microcatheter; RDR: retrograde dissection and re-entry; RWE: retrograde wire escalation.

\section{Antegrade Wire Escalation as First Choice, Contralateral Interventional Collaterals Available for Retrograde Options in Case of Antegrade Wire Escalation Failure}

In this case, the operator decides to start the procedure with the AWE technique using a single 6 Fr sheath in the forearm. The operator starts by using hydrophilic guidewires with low penetration power (e.g., Fielder XT-R; Asahi Intecc Co., Seto, Japan) to pass through the lesion while remaining inside the lumen of the vessel. Sometimes, an appropriate visualisation of the distal vessel is not possible with only a single injection due to the presence of competitive flow between the target and the main vessel. This specific scenario in which the lesion crossing is performed without seeing the distal segment beyond the CTO has been defined as 'blind wiring escalation'. Therefore, the operator should be guided by tactile feedback more than visual feedback.

For this reason, in this specific procedure step, the use of hydrophilic guidewires with intermediateor high-penetration-force that present a scarce tactile feedback is not recommended and should be avoided because of a higher risk of perforation. In case of failure of the blind wiring 
escalation attempt, the operator should switch to a retrograde attempt; in doing so, the operator proceeds to remove the catheter from the CTO vessel and switches it with another guiding catheter to cannulate the contralateral vessel (using the same access as before, thus remaining 'single access'). Afterwards, the donor vessel is engaged in order to cross the collaterals, reach the distal cap of the CTO, and try to face the lesion in a retrograde fashion. If the operator is able to cross the collaterals with the guidewire and the microcatheter, a second 6 Fr sheath is placed in the contralateral wrist and a $6 \mathrm{Fr}$ guiding catheter is used to cannulate the CTO vessel followed by RWE or RDR. If, however, it is not possible to cross the collaterals, a second $7 \mathrm{Fr}$ sheath is placed in the contralateral wrist in order to engage the CTO vessel with a $7 \mathrm{Fr}$ guiding catheter and cross the lesion by means of AWE technique, using guidewires with greater penetrative powers and the double injection, or with ADR.

\section{Antegrade Wire Escalation as First Choice with a Lack of Contralateral Interventional Collaterals for Retrograde Options}

When the operator decides to start the procedure using the AWE technique but no contralateral interventional collaterals for the retrograde approach are available, a single 6 Fr sheath transwrist is used. First, an antegrade attempt with low-penetration-power guidewires is completed in a 'blind wiring' fashion. In case of failure, the operator switches to more advanced antegrade techniques such as the AWE with guidewires with greater penetrative force or ADR. To do so, the $6 \mathrm{Fr}$ guiding is changed for a guiding that fits the donor artery and a second $7 \mathrm{Fr}$ sheath is placed in the contralateral wrist to engage the CTO vessel with a 7 Fr guiding catheter. The original 6 Fr access is used to place a 6 Fr guiding catheter in the donor vessel in order to perform the contralateral injection and obtain a better visualisation of the distal target vessel anatomy (double injection), which is mandatory to make the procedure safer.

\section{Retrograde Wire Escalation/ Retrograde Dissection and Re- entry When Contralateral Collaterals}

\section{Represent the Main Source of Interventional Collaterals}

In this approach a single wrist access with $6 \mathrm{Fr}$ sheath is recommended as the initial set-up to cross the contralateral collaterals. If the operator is able to reach the distal segment of the target vessel via collaterals with a guidewire and microcatheter, a second $6 \mathrm{Fr}$ sheath is placed in the contralateral wrist to finalise the retrograde strategy (RWE/RDR). If it is not possible to cross the collaterals, antegrade advanced techniques remain the last options (AWE with intermediateor high-penetrative-force guidewires or ADR) and a guiding catheter with more internal space is needed. Thus, a second 7 Fr sheath should be inserted into the contralateral forearm in order to place a $7 \mathrm{Fr}$ guiding catheter in the CTO vessel.

\section{Antegrade Wire Escalation Without Interventional Collaterals}

The set-up in the case of ADR consists of double trans-wrist access ( $7 \mathrm{Fr}$ and $6 \mathrm{Fr}$ introducer sheath) because of the need for simultaneous double injection for clear visualisation of the target vessel course. A $7 \mathrm{Fr}$ guiding catheter is placed in the CTO vessel, while a $6 \mathrm{Fr}$ guiding catheter is inserted in the donor vessel for contralateral injection. All of the different techniques (AWE, RWE, RDR) are possible in case of ADR failure.

\section{Any Antegrade or Retrograde Approach in Case of Ipsilateral Collaterals}

When ipsilateral collaterals are the main source of collateral visualisation, the insertion of a single $7 \mathrm{Fr}$ introducer sheath (trans-wrist) is suggested. This set-up allows the use of all four techniques. However, when the operator fails to cross the lesion with a single catheter, a second 6 Fr sheath can be placed in the other wrist to add a second catheter in the same coronary vessel and allow 'ping-pong' techniques.

\section{STRENGTHS AND WEAKNESSES OF THE 'MINIMALISTIC HYBRID APPROACH' ALGORITHM}

The advantage of the modified hybrid algorithm is represented by the minimisation of 
double access, sheath size, and trans-femoral approaches, potentially reducing the number of vascular-related complications as well as the discomfort for the patient. However, some critical issues have been raised.

\section{The Use of Blind Wiring Technique}

Blind wiring has been considered the main weakness of the algorithm because it does not follow one of the 'commandments' of CTO operators: the simultaneous double injection. According to some expert opinions, the lack of dual injection could make the procedure less safe due to poor angiographic visualisation and consequent increased risk of perforation and tamponade. However, by using soft guidewires in this particular setting, it is possible to tackle the lesion without increasing this risk of perforation. A recent publication showed the safety of a single-catheter approach in a small group of patients with СTO undergoing percutaneous revascularisation. ${ }^{17}$

\section{Obtaining Second Access in the Middle of the Intervention}

The need to obtain second access during the procedure, after the patient has already received a full dose of heparin, could impose a higher risk of access site bleeding. However, a strategy to minimise this risk is to use forearm approach or use ultrasound guidance when femoral access is needed.

\section{The Use of Small-Bore Catheters}

In some scenarios a 6-7 Fr catheter could be inappropriate because of the lack of enough support. To overcome this drawback, the authors recommend the use of supportive catheters such as the Amplatz left catheter for the right coronary artery or an oversized extra back-up catheter for the left coronary artery. Additional tools such as guide extension devices should be used with low threshold in case of inadequate support.

It must be noted that the use of single-access and small catheters make the CTO procedure, which is already challenging when approached with standard techniques, even more complex.
Because of this, the MHA is aimed for expert СTO interventional cardiologists, with deep knowledge of material properties, awareness of the potential drawbacks, and long experience in the use of the classic hybrid algorithm as well as forearm access.

\section{EFFICACY AND SAFETY OF THE 'MINIMALISTIC HYBRID APPROACH' ALGORITHM}

The efficacy and safety of this new approach have been recently evaluated in one retrospective and one prospective single-centre study. In the former, a group of 91 patients were treated with the MHA algorithm. The authors observed a success rate of $89.0 \%$, a perforation rate of $2.2 \%$, and no vascular complications. Interestingly, in $52(64.2 \%)$ cases there was no need for a second access to achieve procedural success. Finally, when compared with the conventional treatment, despite the small number of patients in the classic hybrid approach group, no differences were found between the two approaches with respect to successful revascularisation. ${ }^{18}$ These results have been confirmed in a consecutive series of $56 \mathrm{CTO}-\mathrm{PCl}$, showing a success rate of approximately 91\%, achieved with single transwrist access in 33 out of 56 patients (58.9\%). Periprocedural complications occurred in three (5.5\%) cases, with only one perforation and one vascular complication (pseudoaneurysm of distal radial artery).$^{19}$ These studies confirm a low rate of complications comparable with previous large registries.

\section{CONCLUSION}

The MHA algorithm shows encouraging results and may represent a valid alternative to the classic hybrid algorithm, with the advantage of reducing the risk of vascular-related complications without increasing the rate of perforation or affecting the frequency of successful revascularisation. However, further large, multicentre studies are needed to confirm these preliminary results and to evaluate the reproducibility of the algorithm by other CTO operators. 


\section{References}

1. Azzalini $L$ et al. Long-term outcomes of chronic total occlusion recanalization versus percutaneous coronary intervention for complex non-occlusive coronary artery disease. Am J Cardiol. 2020;125(2):182-8.

2. Lee S-W et al. Randomized trial evaluating percutaneous coronary intervention for the treatment of chronic total occlusion. Circulation. 2019;139(14):1674-83.

3. Riley RF et al. Prevalence, predictors, and health status implications of periprocedural complications during coronary chronic total occlusion angioplasty. Eurolntervention. 2018;14(11):e1199-206.

4. Nikolakopoulos I et al. Latest developments in chronic total occlusion percutaneous coronary intervention. Expert Rev Cardiovasc Ther. 2020;18(7):415-26.

5. Morino $Y$ et al. Predicting successfu guidewire crossing through chronic total occlusion of native coronary lesions within 30 minutes: the J-CTO (multicenter CTO registry in Japan) score as a difficulty grading and time assessment tool. JACC CardiovasC Interv. 2011;4(2):213-21.

6. Christopoulos $\mathrm{G}$ et al. Application and outcomes of a hybrid approach to chronic total occlusion percutaneous coronary intervention in a contemporary multicenter US registry. Int J Cardiol. 2015;198:222-8.

7. Brilakis ES et al. A percutaneous treatment algorithm for crossing coronary chronic total occlusions.

JACC Cardiovasc Interv.

2012;5(4):367-79.

8. Vescovo GM et al. Further advancement in the percutaneous revascularization of coronary chronic total occlusions: the redefined "minimalistic hybrid approach" algorithm. Minerva Cardioangiol. 2020;DOI:10.23736/SOO26 4725.20.05381-5.

9. Bufe $A$ et al. Recanalisation of coronary chronic total occlusions with new techniques including the retrograde approach via collaterals. Neth Heart J. 2011;19(4):162-7.

10. Dash D. Retrograde coronary chronic total occlusion intervention. Curr Cardiol Rev. 2015;11(4):291-8.

11. Di Mario C, Bambagioni G. Coronary collaterals, the natural grafts of CTO lesions - good enough to keep most myocardium alive, unable to prevent ischaemia. Eurolntervention. 2020;16(6):e441-4.

12. Matsuno S et al. Overview and proposed terminology for the reverse controlled antegrade and retrograde tracking (reverse CART) techniques. Eurolntervention. 2018;14(1):94-101.

13. Sapontis J et al. Early procedural and health status outcomes after chronic total occlusion angioplasty: a report from the OPEN-CTO registry (outcomes, patient health status, and efficiency in chronic total occlusion hybrid procedures). JACC Cardiovasc Interv. 2017;10(15):1523-34.
14. Singh $\mathrm{M}$ et al. Utility of bilatera coronary injections during complex coronary angioplasty. J Invasive Cardiol. 1999;11(2):70-4.

15. Zivelonghi J.P et al. Implementing a minimally invasive approach (combining radial approach, small guiding catheters and minimization of double access) for coronary chronic total occlusion intervention according to the hybrid algorithm: the minimalistic hybrid algorithm. Int J Cardiol. 2019;283:84-7.

16. Tajti P et al. Procedural outcomes of percutaneous coronary interventions for chronic total occlusions via the radial approach: insights from an international chronic total occlusion registry. JACC Cardiovasc Interv. 2019;12(4):346-58

17. Alaswad $\mathrm{K}$ et al. Transradial approach for coronary chronic total occlusion interventions: Insights from a contemporary multicenter registry. Catheter Cardiovasc Interv. 2015;85(7):1123-9.

18. Zivelonghi $\mathrm{C}$ et al. A "minimalistic hybrid algorithm" in coronary chronic total occlusion revascularization: procedural and clinical outcomes. Catheter Cardiovasc Interv. 2020;95(1):97-104

19. Agostoni $\mathrm{P}$ et al. A prospective evaluation of a redefined version of the "minimalistic hybrid approach" algorithm for percutaneous coronary chronic total occlusion revascularization. Catheter Cardiovasc Interv. 2020;DOI:10.1002/ ccd. 29158. 